\title{
Application of the Adomian Decomposition Method to the Solution of the Linear Nonhomogeneous One-Dimensional Wave Equation
}

\author{
Christian Kasumo \\ Department of Science and Mathematics, Mulungushi University, P.O. Box 80415, Kabwe, Zambia.
}

\begin{abstract}
How to cite this paper: Christian Kasumo. (2020) Application of the Adomian Decomposition Method to the Solution of the Linear Nonhomogeneous One-Dimensional Wave Equation. Journal of Applied Mathematics and Computation, 4(2), 34-42. DOI: 10.26855/jamc.2020.06.004
\end{abstract}

Received: June 8, 2020

Accepted: June 30, 2020

Published: July 7, 2020

*Corresponding author: Christian Kasumo, Department of Science and Mathematics, Mulungushi University, P.O. Box 80415, Kabwe, Zambia.

Email: ckasumo@mu.ac.zm

\begin{abstract}
The Adomian decomposition method is employed in the solution of the linear nonhomogeneous one-dimensional wave equation. This method leads to an analytical solution in the form of an infinite power series. A comparison of the results obtained by the numerical method with the exact solution from d'Alembert's formula shows the high degree of accuracy of the Adomian decomposition method.
\end{abstract}

\section{Keywords}

Nonhomogeneous wave equation, Adomian decomposition method, D’Alembert's formula

\section{Motivation}

The wave equation is one of three fundamental equations in mathematical physics, the other two being the heat and Laplace equations. These equations occur in applied mathematics, engineering and many branches of physics. There are two types of these equations: The homogeneous equations that have constant coefficients with many classical solutions such as the separation of variables, the method of characteristics, the single Laplace transform and the Fourier transform [1-5] and the nonhomogeneous equations with constant coefficients solved by means of the double Laplace transform and operation calculus [6, 7].

The linear homogeneous one-dimensional wave equation has been solved using the Adomian decomposition method (ADM) in Kasumo [8] where the merits and demerits of the ADM have been beautifully outlined. This paper seeks to extend [8] by applying the ADM to obtain the exact or near-exact solutions to the linear nonhomogeneous one-dimensional wave equation. The concern here is how to deal with the source term and hopefully set the stage for solving the nonlinear wave equation. Consider the following basic nonhomogeneous setup for the wave equation:

$$
\left\{\begin{array}{c}
u_{t t}-c^{2} u_{x x}=F(x, t) \\
u\left(x, t_{0}\right)=f(x) \\
u_{t}\left(x, t_{0}\right)=g(x)
\end{array}\right.
$$

where $F(x, t), f(x)$ and $g(x)$ are sufficiently smooth known functions. In this work, we use the Adomian series solution method which yields the exact solution of problem (1). The rest of the paper is organised as follows: Section 2 outlines the method of solution, Section 3 gives a sampling of numerical examples and Section 4 concludes the study. 


\section{Adomian Decomposition Method}

\subsection{Operator form}

In this section, we outline the steps to obtain a solution of (1) using the ADM which was formulated by Adomian [9-11]. In the standard form, (1) can be rewritten as:

$$
L_{t}(u(x, t))=L_{x}\left(c^{2} u(x, t)\right)+F(x, t)
$$

Where the differential operators are defined, respectively, as $L_{t}=\frac{\partial^{2}}{\partial t^{2}}$ and $L_{x}=\frac{\partial^{2}}{\partial x^{2}}$. Suppose the inverse operator $L_{t}^{-1}$ exists. Then $L_{t}^{-1}$ is the two-fold integral operator from 0 to $t$ defined by:

$$
L_{t}^{-1}=\int_{0}^{t} \int_{0}^{t}(.) d s d s
$$

Applying this inverse operator on both sides of equation (2) gives

$$
L_{t}^{-1}\left[L_{t}(u(x, t))\right]=L_{t}^{-1}\left[L_{x}\left(c^{2} u(x, t)\right)\right]+L_{t}^{-1}[F(x, t)]
$$

Since $c^{2}$ is a constant, it can be factored out and the above equation can be written as:

$$
L_{t}^{-1}\left[L_{t}(u(x, t))\right]=c^{2} L_{t}^{-1}\left[L_{x}(u(x, t))\right]+L_{t}^{-1}[F(x, t)]
$$

\subsection{Application of the ADM to the solution of the problem}

From equation (3), it follows that

$$
u(x, t)=A+B t+c^{2} L_{t}^{-1}\left[L_{x}(u(x, t))\right]+L_{t}^{-1}[F(x, t)]
$$

Where $A$ and $B$ are constants of integration which can be found from the boundary or initial conditions. Assuming that $t_{0}=0$ in the given initial conditions, $A=f(x)$ and $B=g(x)$, so that we have

$$
u(x, t)=f(x)+\operatorname{tg}(x)+c^{2} L_{t}^{-1}\left[L_{x}(u(x, t))\right]+L_{t}^{-1}[F(x, t)]
$$

Where the unknown function $u$ is decomposed into a sum of components defined by the decomposition series:

$$
u(x, t)=\sum_{n=0}^{\infty} u_{n}(x, t)
$$

with $u_{0}$ identified as $u(x, 0)$ and the components $u_{n}(x, t)$ obtained from the recursive formula:

$$
\sum_{n=0}^{\infty} u_{n}(x, t)=f(x)+\operatorname{tg}(x)+c^{2} L_{t}^{-1}\left[L_{x}\left(\sum_{n=0}^{\infty} u_{n}(x, t)\right)\right]+L_{t}^{-1}[F(x, t)]
$$

or

i.e.,

$$
\begin{aligned}
& u_{0}(x, t)=f(x)+\operatorname{tg}(x)+L_{t}^{-1}[F(x, t)] \\
& u_{n+1}(x, t)=c^{2} L_{t}^{-1}\left[L_{x}(u(x, t))\right], n \geq 0
\end{aligned}
$$

$$
\begin{gathered}
u_{0}=f(x)+\operatorname{tg}(x)+L_{t}^{-1}[F(x, t)] \\
u_{1}=c^{2} L_{t}^{-1}\left[L_{x}\left(u_{0}\right)\right] \\
u_{2}=c^{2} L_{t}^{-1}\left[L_{x}\left(u_{1}\right)\right] \\
\vdots \\
u_{n+1}=c^{2} L_{t}^{-1}\left[L_{x}\left(u_{n}\right)\right]
\end{gathered}
$$

Thus, the ADM is concerned with finding the components $u_{0}, u_{1}, u_{2}, \ldots$ individually and summing them up to obtain the solution $u(x, t)$. If the PDE contains a nonlinear term, this term is represented as $N u$ and decomposed into a series:

$$
N u=\sum_{n=0}^{\infty} A_{n}
$$

Where the $A_{n}$, depending on $u_{0}, u_{1}, u_{2}, \ldots, u_{n}$, are called Adomian polynomials obtained, for the nonlinearity $N u=\beta(u)$, by the formula: 


$$
A_{n}=\frac{1}{n !} L_{\lambda}\left[\beta\left(\sum_{i=0}^{\infty} u_{i} \lambda^{i}\right)\right]
$$

Where $\lambda$ is a grouping parameter of convenience and $L_{\lambda}=\frac{\partial^{n}}{\partial \lambda^{n}}$. In this way, the components $u_{0}, u_{1}, u_{2}, \ldots$ are identified and the series solution to the wave equation is completely determined. The exact solution may be determined using the approximation:

Where $\Phi_{n}=\sum_{k=0}^{n-1} u_{k}$.

$$
u(x, t)=\lim _{n \rightarrow \infty} \Phi_{n}
$$

Remark 2.1. Note that the recursive relationship (7) is constructed on the basis that the zeroth component $u_{0}(x, t)$ is defined by all the terms that arise from the initial conditions and from integrating the source term. The remaining components $u_{n}(x, t), n \geq 1$ are completely determined recursively such that each term is computed by using the immediately preceding term. Accordingly, considering the first few terms only, the recursive relation (7) gives $u_{0}(x, t), u_{1}(x, t), u_{2}(x, t), \ldots$

Remark 2.2. Adomian and Rach [11] and Wazwaz [12] investigated the phenomenon of self-cancelling "noise" terms where some terms in the series vanish in the limit. These "noise" terms do not appear for homogeneous equations but only for specific types of nonhomogeneous equations. Furthermore, it has formally been shown that if some terms in $u_{0}$ are cancelled by identical terms in $u_{1}$ with opposite signs, even though $u_{1}$ includes additional terms, the remaining non-cancelled terms in $u_{0}$ constitute the exact solution of the given equation (Kaya and Inc [13]; Almazmumy et al. [14]). However, it is necessary and essential to verify that the remaining non-cancelled terms satisfy the given equation.

\subsection{D’Alembert's formula for the nonhomogeneous wave equation}

Given certain regularity, the unique analytical solution of the linear nonhomogeneous one-dimensional wave equation is given by a general form of d'Alembert's formula according to the following theorem.

Theorem 2.3. Consider the nonhomogeneous wave equation given in (1). Fix $k \geq 1$ and taket $t_{0} \in\left(T_{-}, T_{+}\right)$. If $f \in$ $C^{k+1}(\mathbb{R}), g \in C^{k}(\mathbb{R}), F \in C^{k}\left[\left(T_{-}, T_{+}\right) \times \mathbb{R}\right]$, then $\exists$ a unique solution $u \in C^{k+1}\left[\left(T_{-}, T_{+}\right) \times \mathbb{R}\right]$ given by:

$$
u(x, t)=\frac{1}{2} f\left(x-c\left(t-t_{0}\right)\right)+\frac{1}{2} f\left(x+c\left(t-t_{0}\right)\right)+\frac{1}{2 c} \int_{x-c\left(t-t_{0}\right)}^{x+c\left(t-t_{0}\right)} g(s) d s+\frac{1}{2 c} \int_{t_{0}}^{t}\left[\int_{x-c(t-s)}^{x+c(t-s)} F(y, s) d y\right] d s
$$

Proof. See Hopkins [15] for proof of the case $c=1$.

Remark 2.4. If $t_{0}=0$, then (8) becomes:

$$
u(x, t)=\frac{1}{2} f(x-c t)+\frac{1}{2} f(x+c t)+\frac{1}{2 c} \int_{x-c t}^{x+c t} g(s) d s+\frac{1}{2 c} \int_{0}^{t}\left[\int_{x-c(t-s)}^{x+c(t-s)} F(y, s) d y\right] d s
$$

Which is the solution of the nonhomogeneous Cauchy wave problem. The first two terms of (9) come from d'Alembert's formula for the homogeneous wave equation (see Kasumo [8]). The third term is called Duhamel's principle and generally works for linear differential equations. Thus, (9) is the solution of the wave equation (1) with $t_{0}=0$. Using the linearity of this equation, we divide it into two smaller sub-problems, namely,

$$
v_{t t}-c^{2} v_{x x}=0, v(x, 0)=f(x), v_{t}(x, 0)=g(x)
$$

and

and solve them separately.

$$
w_{t t}-c^{2} w_{x x}=F(x, t), w(x, 0)=0, w_{t}(x, 0)=0
$$

Lemma 2.5. Let $v$ be the solution of (10) and $w$ the solution of (11). Then $u=v+w$ is the solution of (1) when $t_{0}=0$, and this solution happens to be (9).

\section{Numerical Examples}

In this section, we give numerical examples of linear nonhomogeneous wave equations and show their solution using the ADM. The examples show that in most cases the exact solution in closed form can be obtained. All the computations associated with these examples were performed using a Samsung Series 3 PC with an Intel Celeron CPU 847 at 1.10GHz and 6.0GB internal memory. The figures were constructed using MATLAB R2016a.

Example 3.1. Consider the nonhomogeneous wave equation

$$
\begin{aligned}
& u_{t t}-u_{x x}=1,-\infty \leq x \leq \infty, t>0 \\
& u(x, 0)=x^{2}
\end{aligned}
$$




$$
u_{t}(x, 0)=1
$$

We use d'Alembert's formula (Theorem 2.3, equation (9)) with $c=1, F(x, t)=1, f(x)=x^{2}$ and $g(x)=1$. Thus, we have

1) $\frac{1}{2} f(x-c t)+\frac{1}{2} f(x+c t)=\frac{1}{2}\left[(x-t)^{2}+(x+t)^{2}\right]=x^{2}+t^{2}$

2) $\frac{1}{2 c} \int_{x-c t}^{x+c t} g(s) d s=\frac{1}{2} \int_{x-t}^{x+t} d s=\frac{1}{2}[(x+t)-(x-t)]=t$

3) $\frac{1}{2 c} \int_{0}^{t}\left[\int_{x-c(t-s)}^{x+c(t-s)} F(y, s) d y\right] d s=\frac{1}{2} \int_{0}^{t} \int_{x-t+s}^{x+t-s} d y d s=\int_{0}^{t}(t-s) d s=t^{2}-\frac{t^{2}}{2}=\frac{t^{2}}{2}$

Adding these three quantities gives the exact solution as,

$$
u(x, t)=x^{2}+t^{2}+t+\frac{t^{2}}{2}=x^{2}+\frac{3 t^{2}}{2}+t
$$

The numerical solution obtained by the ADM will be compared with the exact solution (12). The ADM solution is given by the recursive relationship,

$$
\begin{gathered}
u_{0}(x, t)=f(x)+t g(x)+L_{t}^{-1}[F(x, t)] \\
u_{n+1}(x, t)=c^{2} L_{t}^{-1}\left[L_{x}\left(\sum_{n=0}^{\infty} u_{n}\right)\right]=c^{2} L_{t}^{-1}\left[\left(\sum_{n=0}^{\infty} u_{n}\right)_{x x}\right]
\end{gathered}
$$

i.e.,

$$
\begin{aligned}
& u_{0}=x^{2}+t+L_{t}^{-1}[1]=x^{2}+t+\int_{0}^{t} \int_{0}^{t} d s d s=x^{2}+t+\int_{0}^{t} s d s=x^{2}+t+\frac{t^{2}}{2} \\
& u_{1}=L_{t}^{-1}\left[L_{x}\left(u_{0}\right)\right]=L_{t}^{-1}\left[L_{x}\left(x^{2}+t+\frac{t^{2}}{2}\right)\right]=L_{t}^{-1}[2]=\int_{0}^{t} \int_{0}^{t} 2 d s d s=t^{2} \\
& u_{2}=L_{t}^{-1}\left[L_{x}\left(u_{1}\right)\right]=L_{t}^{-1}\left[L_{x}\left(t^{2}\right)\right]=0=u_{n+1}, n \geq 2
\end{aligned}
$$

Thus, the ADM solution is

$$
u(x, t)=u_{0}+u_{1}+u_{2}=x^{2}+\frac{3 t^{2}}{2}+t
$$

which is the exact solution (equation (12)) obtained by d'Alembert's formula for the nonhomogeneous wave equation (9). The results for $0<x<3$ and $0<t<2$ are given in Figure 1 .

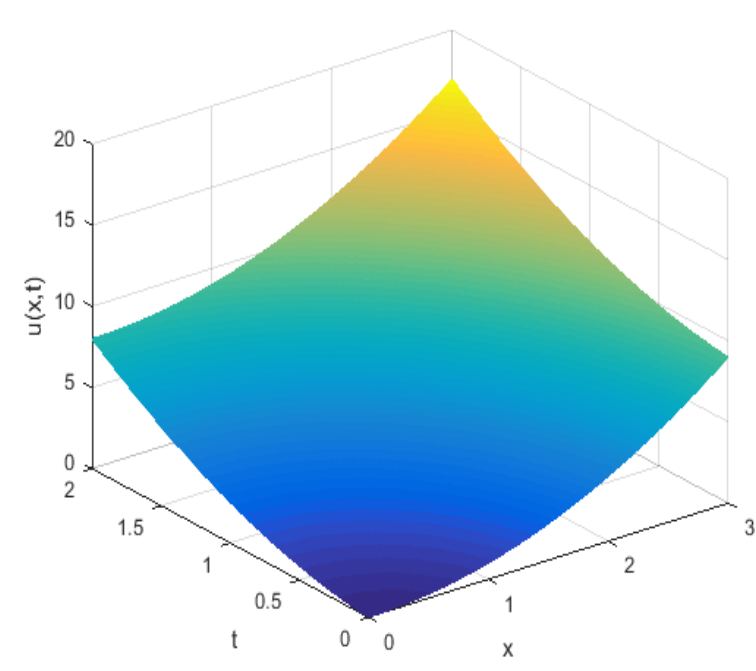

(a) Surface $u(x, t)=x^{2}+\frac{3 t^{2}}{2}+t$

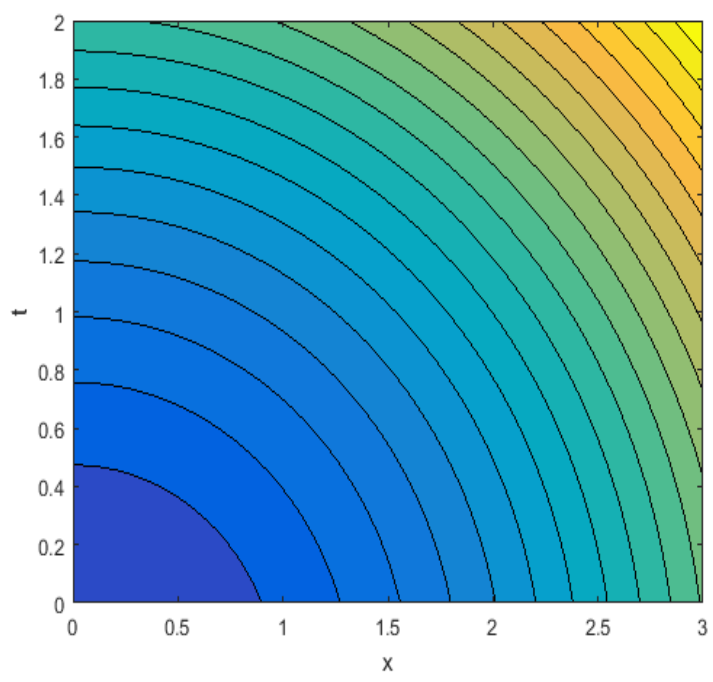

(b) Contour diagram

Figure 1. Approximate and exact solution and contour diagram for Example 3.1.

Example 3.2. Consider the following one-dimensional nonhomogeneous wave equation: 


$$
\begin{aligned}
& u_{t t}-u_{x x}=t \sin x,-\infty \leq x \leq \pi, t>0 \\
& u(x, 0)=\sin x \\
& u_{t}(x, 0)=\sin 3 x
\end{aligned}
$$

Here, $c=1, F(x, t)=t \sin x, f(x)=\sin x$ and $g(x)=\sin 3 x$. Thus,

1) $\frac{1}{2} f(x-c t)+\frac{1}{2} f(x+c t)=\frac{1}{2}[\sin (x-t)+\sin (x+t)]=\sin x \cos t$

2) $\frac{1}{2 c} \int_{x-c t}^{x+c t} g(s) d s=\frac{1}{2} \int_{x-t}^{x+t} \sin 3 s d s=-\frac{1}{6}[\cos (3 x+3 t)-\cos (3 x-3 t)]=\frac{1}{3} \sin 3 x \sin 3 t$

3) $\frac{1}{2 c} \int_{0}^{t}\left[\int_{x-c(t-s)}^{x+c(t-s)} F(y, s) d y\right] d s=\frac{1}{2} \int_{0}^{t} \int_{x-t+s}^{x+t-s} s \sin y d y d s=\int_{0}^{t} s \sin x \sin (t-s) d s=(t-\sin t) \sin x$

By d'Alembert's formula, the exact solution is the sum of the three quantities obtained above:

$$
u(x, t)=\sin x \cos t+\frac{1}{3} \sin 3 x \sin 3 t+(t-\sin t) \sin x
$$

Now, applying the concept of ADM to the given wave equation, we have the recursive relationship

i.e.,

$$
\begin{gathered}
u_{0}=\sin x+t \sin 3 x+L_{t}^{-1} \\
L_{t}^{-1}\left[\left(\sum_{n=0}^{\infty} u_{n}\right)_{x x}\right], n \geq 0
\end{gathered}
$$

$u_{0}=\sin x+t \sin 3 x+\int_{0}^{t} \int_{0}^{t} s \sin x d s d s=\sin x+t \sin 3 x+\int_{0}^{t} \frac{s^{2}}{2} \sin x d s=\sin x+t \sin 3 x+\frac{t^{3}}{6} \sin x$

$$
\begin{aligned}
& u_{1}=L_{t}^{-1}\left[L_{x}\left(u_{0}\right)\right]=L_{t}^{-1}\left[L_{x}\left(\sin x+t \sin 3 x+\frac{t^{3}}{6} \sin x\right)\right]=L_{t}^{-1}\left[-\sin x-9 t \sin 3 x-\frac{t^{3}}{6} \sin x\right] \\
& =\int_{0}^{t} \int_{0}^{t}\left[-\sin x-9 t \sin 3 x-\frac{s^{3}}{6} \sin x\right] d s d s=\int_{0}^{t}\left[-s \sin x-\frac{9 s^{2}}{2} \sin 3 x-\frac{s^{4}}{24} \sin x\right] d s \\
& =-\frac{t^{2}}{2} \sin x-\frac{9 t^{3}}{6} \sin 3 x-\frac{t^{5}}{120} \sin x \\
& u_{2}=L_{t}^{-1}\left[L_{x}\left(u_{1}\right)\right]=L_{t}^{-1}\left[L_{x}\left(-\frac{t^{2}}{2} \sin x-\frac{9 t^{3}}{6} \sin 3 x-\frac{t^{5}}{120} \sin x\right)\right]=L_{t}^{-1}\left[\frac{t^{2}}{2} \sin x+\frac{81 t^{3}}{6} \sin 3 x+\frac{t^{5}}{120} \sin x\right]
\end{aligned}
$$$$
=\int_{0}^{t} \int_{0}^{t}\left[\frac{s^{2}}{2} \sin x+\frac{81 s^{3}}{6} \sin 3 x+\frac{s^{5}}{120} \sin x\right] d s d s=\int_{0}^{t}\left[\frac{s^{3}}{6} \sin x+\frac{81 s^{4}}{24} \sin 3 x+\frac{s^{6}}{720} \sin x\right] d s
$$$$
=\frac{t^{4}}{24} \sin x+\frac{81 t^{5}}{120} \sin 3 x+\frac{t^{7}}{5040} \sin x
$$

and so on. Adding the above approximants gives the solution as, $u=u_{0}+u_{1}+u_{2}+\cdots$

i.e.,

$$
\begin{gathered}
=\sin x+t \sin 3 x+\frac{t^{3}}{6} \sin x-\frac{t^{2}}{2} \sin x-\frac{9 t^{3}}{6} \sin 3 x-\frac{t^{5}}{120} \sin x+\frac{t^{4}}{24} \sin x+\frac{81 t^{5}}{120} \sin 3 x+\frac{t^{7}}{5040} \sin x+\cdots \\
=\sin x-\frac{t^{2}}{2} \sin x+\frac{t^{4}}{24} \sin x+\cdots+t \sin 3 x-\frac{9 t^{3}}{6} \sin 3 x+\frac{81 t^{5}}{120} \sin 3 x+\cdots+\frac{t^{3}}{6} \sin x-\frac{t^{5}}{120} \sin x \\
\quad+\frac{t^{7}}{5040} \sin x+\cdots \\
=\sin x\left(1-\frac{t^{2}}{2}+\frac{t^{4}}{24}-\cdots\right)+\frac{1}{3} \sin 3 x\left(3 t-\frac{27 t^{3}}{6}+\frac{243 t^{5}}{120}-\cdots\right)+\sin x\left(\frac{t^{3}}{6}-\frac{t^{5}}{120}+\frac{t^{7}}{5040}-\cdots\right) \\
=\sin x\left(1-\frac{t^{2}}{2 !}+\frac{t^{4}}{4 !}-\cdots\right)+\frac{1}{3} \sin 3 x\left(3 t-\frac{(3 t)^{3}}{3 !}+\frac{(3 t)^{5}}{5 !}-\cdots\right)+\sin x\left(\frac{t^{3}}{3 !}-\frac{t^{5}}{5 !}+\frac{t^{7}}{7 !}-\cdots\right)
\end{gathered}
$$

$$
u(x, t)=\sin x \cos t+\frac{1}{3} \sin 3 x \sin 3 t+(t-\sin t) \sin x
$$


which is the exact solution obtained from d'Alembert's formula. Figure 2 gives the results for $0<x<3$ and $0<t<2$.

Example 3.3. Consider the following Cauchy problem of the nonhomogeneous wave equation,

$$
\begin{aligned}
& u_{t t}-u_{x x}=t^{7},-\infty \leq x \leq \infty, t>0 \\
& u(x, 0)=2 x+\sin x \\
& u_{t}(x, 0)=0
\end{aligned}
$$

To find the solution, we first use d'Alembert's formula with $c=1, F(x, t)=t^{7}, f(x)=2 x+\sin x$ and $g(x)=0$.

1) $\frac{1}{2} f(x-c t)+\frac{1}{2} f(x+c t)=\frac{1}{2}[2(x-t)+\sin (x-t)+2(x+t)+\sin (x+t)]=2 x+\sin x \cos t$

2) $\frac{1}{2 c} \int_{x-c t}^{x+c t} g(s) d s=0$

3) $\frac{1}{2 c} \int_{0}^{t}\left[\int_{x-c(t-s)}^{x+c(t-s)} F(y, s) d y\right] d s=\frac{1}{2} \int_{0}^{t} \int_{x-t+s}^{x+t-s} s^{7} d y d s=\frac{1}{2} \int_{0}^{t}[2 t-2 s] s^{7} d s=\int_{0}^{t}\left[t s^{7}-s^{8}\right] d s=\frac{t^{9}}{8}-\frac{t^{9}}{9}=\frac{t^{9}}{72}$
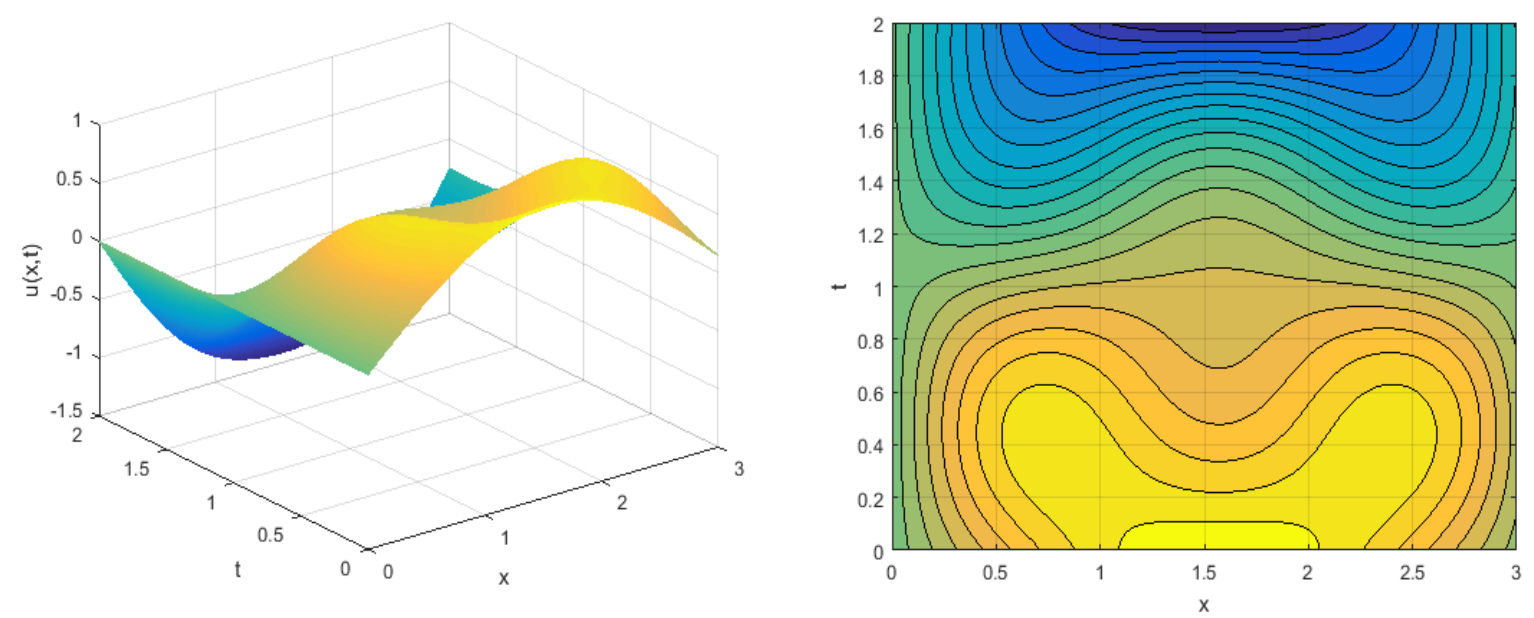

(a) Surface $u(x, t)=\sin x \cos t+\frac{1}{3} \sin 3 x \sin 3 t+(t-\sin t) \sin x$

(b) Contour diagram

Figure 2. Approximate and exact solution and contour diagram for Example 3.2.

The analytical solution is therefore,

$$
u(x, t)=2 x+\sin x \cos t+\frac{t^{9}}{72}
$$

By the ADM, we have, recursively, the approximants:

$$
\begin{gathered}
u_{0}=2 x+\sin x+L_{t}^{-1}\left[t^{7}\right]=2 x+\sin x+\int_{0}^{t} \int_{0}^{t} s^{7} d s d s=2 x+\sin x+\frac{t^{9}}{72} \\
u_{1}=L_{t}^{-1}\left[L_{x}\left(u_{0}\right)\right]=L_{t}^{-1}\left[L_{x}\left(2 x+\sin x+\frac{t^{9}}{72}\right)\right]=L_{t}^{-1}[-\sin x]=\int_{0}^{t} \int_{0}^{t}-\sin x d s d s=-\frac{t^{2}}{2} \sin x \\
u_{2}=L_{t}^{-1}\left[L_{x}\left(u_{1}\right)\right]=L_{t}^{-1}\left[L_{x}\left(-\frac{t^{2}}{2} \sin x\right)\right]=L_{t}^{-1}\left[\frac{t^{2}}{2} \sin x\right]=\int_{0}^{t} \int_{0}^{t} \frac{s^{2}}{2} \sin x d s d s=\frac{t^{4}}{24} \sin x \\
u_{3}=L_{t}^{-1}\left[L_{x}\left(u_{2}\right)\right]=L_{t}^{-1}\left[L_{x}\left(\frac{t^{4}}{24} \sin x\right)\right]=L_{t}^{-1}\left[-\frac{t^{4}}{24} \sin x\right] \\
=\int_{0}^{t} \int_{0}^{t}-\frac{s^{4}}{24} \sin x d s d s=-\frac{t^{6}}{120} \sin x
\end{gathered}
$$

and so on. Summing up the approximants $u_{0}, u_{1}, u_{3}, \ldots$, we obtain the desired approximate solution: 


$$
\begin{aligned}
u=u_{0}+u_{1}+u_{2} & +\cdots=2 x+\sin x+\frac{t^{9}}{72}-\frac{t^{2}}{2} \sin x+\frac{t^{4}}{24} \sin x-\frac{t^{5}}{120} \sin x+\cdots \\
& =2 x+\sin x\left(1-\frac{t^{2}}{2 !}+\frac{t^{4}}{4 !}-\frac{t^{6}}{6 !}+\cdots\right)+\frac{t^{9}}{72}
\end{aligned}
$$

i.e.,

$$
u(x, t)=2 x+\sin x \cos t+\frac{t^{9}}{72}
$$

Which is simply the exact solution (16). The results for $0<x<3$ and $0<t<2$ are shown in Figure 3 .

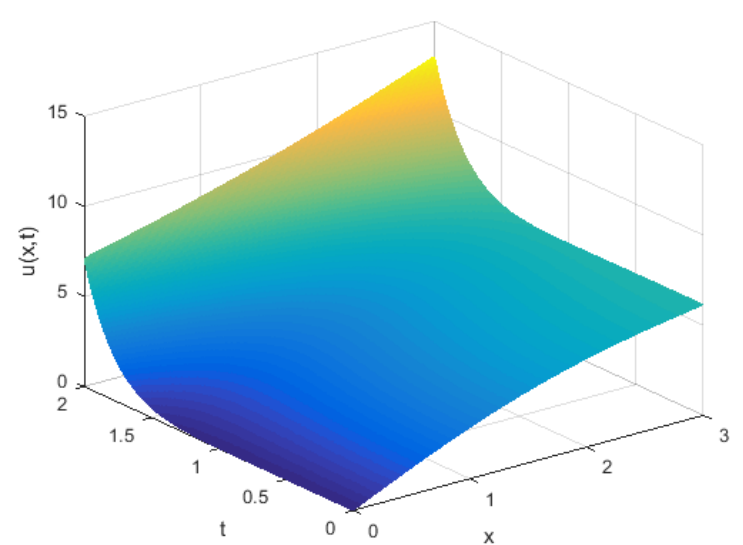

(a) Surface $u(x, t)=2 x+\sin x \cos t+\frac{t^{9}}{72}$

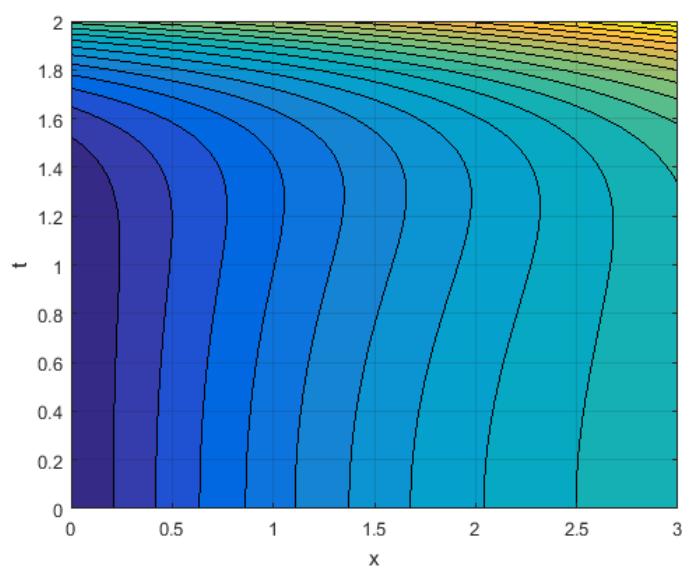

(b) Contour diagram

Figure 3. Approximate and exact solution and contour diagram for Example 3.3.

Example 3.4. Consider the following Cauchy problem of the nonhomogeneous wave equation

$$
\begin{aligned}
& u_{t t}-u_{x x}=1,-\infty \leq x \leq \infty, t>0 \\
& u(x, 0)=\sin x \\
& u_{t}(x, 0)=x
\end{aligned}
$$

To find the solution, we first use d'Alembert's formula with $c=1, F(x, t)=1, f(x)=\sin x$ and $g(x)=x$.

1) $\frac{1}{2} f(x-c t)+\frac{1}{2} f(x+c t)=\frac{1}{2}[\sin (x-t)+\sin (x+t)]=\sin x \cos t$

2) $\frac{1}{2 c} \int_{x-c t}^{x+c t} g(s) d s=\frac{1}{2} \int_{x-t}^{x+t} s d s=\frac{1}{4}\left[(x+t)^{2}-(x-t)^{2}\right]=\frac{1}{4}[4 t x]=t x$

3) $\frac{1}{2 c} \int_{0}^{t}\left[\int_{x-c(t-s)}^{x+c(t-s)} F(y, s) d y\right] d s=\frac{1}{2} \int_{0}^{t} \int_{x-t+s}^{x+t-s} d y d s=\frac{1}{2} \int_{0}^{t}[2 t-2 s] d s=\int_{0}^{t}[t-s] d s=t^{2}-\frac{t^{2}}{2}=\frac{t^{2}}{2}$

Thus, the exact solution to the given Cauchy wave problem is:

$$
u(x, t)=\sin x \cos t+t x+\frac{t^{2}}{2}
$$

and the ADM solution is found as the sum of the approximants:

$$
\begin{gathered}
u_{0}=\sin x+t x+L_{t}^{-1}[1]=\sin x+t x+\int_{0}^{t} \int_{0}^{t} d s d s=\sin x+t x+\frac{t^{2}}{2} \\
u_{1}=L_{t}^{-1}\left[L_{x}\left(u_{0}\right)\right]=L_{t}^{-1}\left[L_{x}\left(\sin x+t x+\frac{t^{2}}{2}\right)\right]=L_{t}^{-1}[-\sin x]=\int_{0}^{t} \int_{0}^{t}-\sin x d s d s=-\frac{t^{2}}{2} \sin x \\
u_{2}=L_{t}^{-1}\left[L_{x}\left(u_{1}\right)\right]=L_{t}^{-1}\left[L_{x}\left(-\frac{t^{2}}{2} \sin x\right)\right]=L_{t}^{-1}\left[\frac{t^{2}}{2} \sin x\right]=\int_{0}^{t} \int_{0}^{t} \frac{s^{2}}{2} \sin x d s d s=\frac{t^{4}}{24} \sin x \\
u_{3}=L_{t}^{-1}\left[L_{x}\left(u_{2}\right)\right]=L_{t}^{-1}\left[L_{x}\left(\frac{t^{4}}{24} \sin x\right)\right]=L_{t}^{-1}\left[-\frac{t^{4}}{24} \sin x\right]=\int_{0}^{t} \int_{0}^{t}-\frac{s^{4}}{24} \sin x d s d s=-\frac{t^{6}}{720} \sin x
\end{gathered}
$$


and so on. Adding the approximants gives the approximate solution:

$$
\begin{gathered}
u=u_{0}+u_{1}+u_{2}+\cdots=\sin x+t x+\frac{t^{2}}{2}-\frac{t^{2}}{2} \sin x+\frac{t^{4}}{24} \sin x-\frac{t^{5}}{720} \sin x+\cdots \\
=\sin x\left(1-\frac{t^{2}}{2 !}+\frac{t^{4}}{4 !}-\frac{t^{6}}{6 !}+\cdots\right)+t x+\frac{t^{2}}{2}
\end{gathered}
$$

i.e.,

$$
u(x, t)=\sin x \cos t+t x+\frac{t^{2}}{2}
$$

the same result obtained using d'Alembert's formula. The results are given in Figure 4.

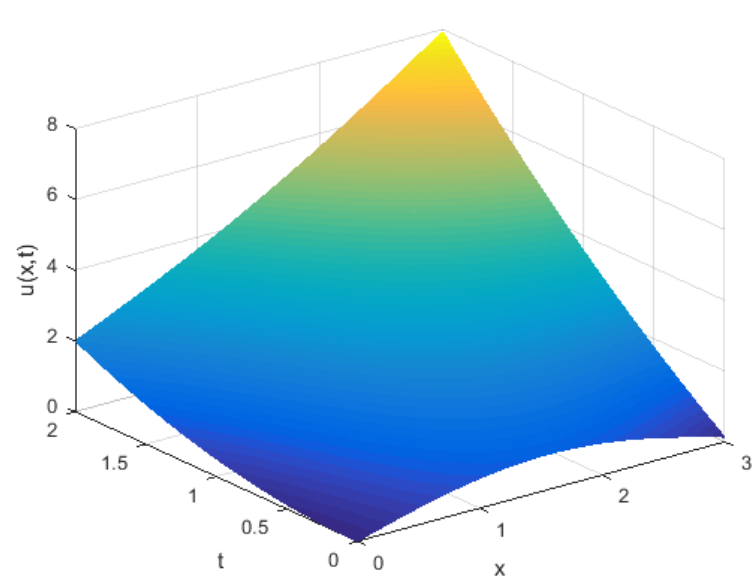

(a) Surface $u(x, t)=\sin x \cos t+t x+\frac{t^{2}}{2}$

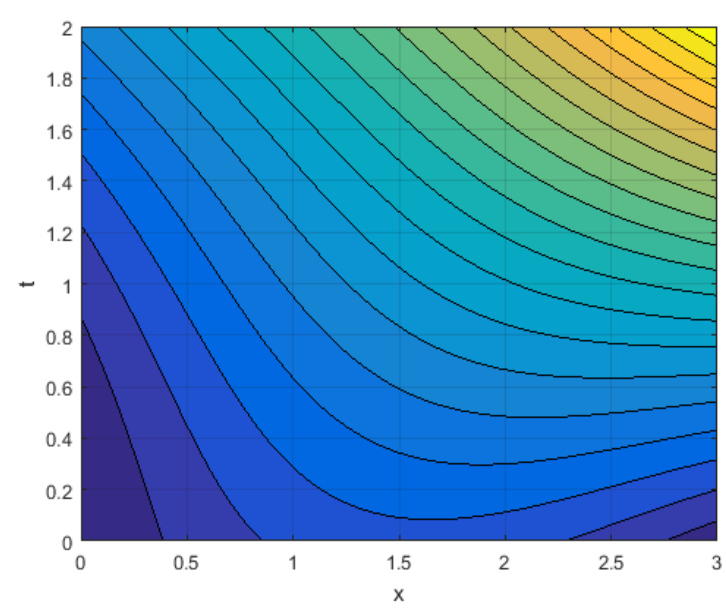

(b) Contour diagram

Figure 4. Approximate and exact solution and contour diagram for Example 3.4.

\section{Conclusion}

In this paper, the Adomian decomposition method has been successfully implemented to find the solution of the linear nonhomogeneous one-dimensional wave equation. Illustrative numerical examples have been given and the results show that the ADM leads to exact solutions to the linear one-dimensional wave equation with a source term. In fact, the ADM can provide analytical or approximate solutions to a wide range of nonlinear and stochastic equations without the need for linearization, perturbation, closure approximation or discretization methods. This work can be improved by (1) using one of the many modifications of the ADM, e.g., Rach et al. [16], Wazwaz [17], Almazmumy et al. [14], Biazar and Hosseini [18] and Alderremy et al. [19]; (2) applying the ADM to the solution of Volterra integro differential and integral equations that frequently occur in insurance mathematics; (3) applying the ADM to partial differential equations with nonlinear terms.

\section{Acknowledgements}

The author wishes to thank Mulungushi University for funding this research and the anonymous referees for their valuable comments which significantly improved the paper.

\section{References}

[1] Lamb Jr., G. L. (1995). Introductory Applications of Partial Differential Equations with Emphasis on Wave Propagation and Diffusion. John Wiley and Sons, New York.

[2] Tyn, M. U. (1980). Partial Differential Equations of Mathematical Physics. Cambridge University Press, Cambridge.

[3] Constanda, C. (2002). Solution Techniques for Elementary Partial Differential Equations. CRC Press, New York.

[4] Duffy, D. G. (2004). Transform Methods for Solving Partial Differential Equations. CRC Press, New York. 
[5] Hu, W. (2017). A New Method to Solve Non-homogeneous Wave Equations of Electromagnetic Fields by Fourier's Triple Integral Transform. Proceedings of 2nd International Conference on Materials Science, Energy Technology and Environmental Engineering, 81, 28-30.

[6] Kiliçman, A. and Eltayeb, H. (2007). A Note on the Non-constant Coefficient Linear Second Order Partial Differential Equation. Proceedings of the Fourth International Conference of Applied Mathematics and Computing, Plovdiv, Bulgaria, 12-18 August, 2007.

[7] Babakhani, A. and Dahiya, R. S. (2001). Systems of Multi-dimensional Laplace Transform and Heat Equation. In: 16th Conference on Applied Mathematics, University of Central Oklahoma, Electronic Journal of Differential Equations Conference, 7 , 25-36.

[8] Kasumo, C. (2019). Solving the Linear Homogeneous One-Dimensional Wave Equation Using the Adomian Decomposition Method. Applied Mathematical Sciences, 13(5), 239-252.

[9] Sun, Z. (2001). A High Order Difference Scheme for a Nonlocal Boundary Value Problem for the Heat Equation. Computational Methods in Applied Mathematics, 1, 398-414.

[10] Adomian, G. (1994). Solving Frontier Problems of Physics: The Decomposition Method. Springer, New York.

[11] Adomian, G. and Rach, R. (1992). Noise Terms in Decomposition Solution Series. Comp. Math. Appl., 24(11), 61-64.

[12] Wazwaz, A.-M. (1997). Necessary Conditions for the Appearance of Noise Terms in Decomposition Solution Series. J. Math. Anal. Appl., 5, 265-274.

[13] Kaya, D. and Inc, M. (1999). On the Solution of the Non-linear Wave Equation by the Decomposition Method. Bull. Malaysian Math. Soc. (second series), 22, 151-155.

[14] Almazmumy, M., Hendi, F. A., Bakodah, H. O. and Alzumi, H. (2012). Recent Modifications of Adomian Decomposition Method for Initial Value Problem in Ordinary Differential Equations. American J. Comput. Math., 2, 228-234.

[15] Hopkins, R. (2016). Nonlinear Wave Equations. University of Chicago, 1-14.

[16] Rach, R., Adomian, G. and Meyers, R. E. (1992). A Modified Decomposition. Comput. Math. Appl., 23, 17-23.

[17] Wazwaz, A.-M. (1999). A Reliable Modification of Adomian Decomposition Method. Appl. Math. Comput., 102, 77-86.

[18] Biazar, J. and Hosseini, K. (2016). A modified Adomian Decomposition Method for Singular Initial Value Emden-Fowler Type Equations. Int. J. Appl. Math. Research, 5(1), 69-72.

[19] Alderremy, A. A., Elzaki, T. M. and Chamekh, M. (2019). Modified Adomian Decomposition Method to Solve Generalized Emden-Fowler Systems for Singular IVP. Mathematical Problems in Engineering, 2019, 1-6. 